\title{
Induction of DNA synthesis and apoptosis are separable functions of E2F-1
}

\author{
Andrew C. Phillips, ${ }^{1}$ Stewart Bates, ${ }^{1}$ Kevin M. Ryan, ${ }^{1}$ Kristian Helin, ${ }^{2}$ and Karen H. Vousden ${ }^{1,3}$ \\ ${ }^{1}$ ABL Basic Research Program, National Cancer Institute-Frederick Cancer Research and Development Center \\ (NCI-FCRDC), Frederick, Maryland 21702 USA; $^{2}$ European Institute of Oncology, Department of Experimental Oncology, \\ 20141 Milan, Italy
}

\begin{abstract}
The family of E2F transcription factors have an essential role in mediating cell cycle progression, and recently, one of the E2F protein family, E2F-1, has been shown to participate in the induction of apoptosis. Cooperation between E2F and the p53 tumor suppressor protein in this apoptotic response had led to the suggestion that cell cycle progression induced by E2F-1 expression provides an apoptotic signal when placed in conflict with an arrest to cell cycle progression, such as provided by $\mathrm{p} 53$. We show here that although apoptosis is clearly enhanced by p53, E2F-1 can induce significant apoptosis in the absence of p53. Furthermore, this apoptotic function of E2F-1 is separable from the ability to accelerate entry into DNA synthesis. Analysis of E2F-1 mutants indicates that although DNA-binding is required, transcriptional transactivation is not necessary for the induction of apoptosis by E2F-1, suggesting that it may be mediated through alleviation of E2F-dependent transcriptional repression. These results indicate that E2F-1 can show independent cell cycle progression and apoptotic functions, consistent with its putative role as a tumor suppressor.
\end{abstract}

[Key Words: E2F; apoptosis; cell cycle; p53]

Received March 11, 1997; revised version accepted June 2, 1997.

Cell cycle progression is a tightly regulated process whose fidelity and ordered progression is monitored at several control points throughout the cycle. Tumor cells frequently show loss of the normal function of these checkpoints, which can lead to unrestrained proliferation and the acquisition of genetic instability (Hartwell and Kastan 1994). Several of the best understood tumor suppressor proteins such as the retinoblastoma gene product $\mathrm{pRB}, \mathrm{p} 53$, and $\mathrm{p} 16$, whose function is frequently lost in many human cancers, play critical roles in maintaining normal cell growth by exerting negative controls on cell cycle progression (Sherr 1996). Although these proteins have multiple functions, one common feature is their ability to target the activity of the E2F family of transcription factors, one of the key mediators of cell cycle progression into S phase (LaThangue 1994). The importance of E2F function has been demonstrated by studies showing that inhibition of E2F prevents entry into S-phase (Wu et al. 1996) and that ectopic expression of E2F in quiescent cells is sufficient for progression into DNA synthesis (Johnson et al. 1993). Active E2F consists of a heterodimer between one of the E2F proteins (E2F15) and a DP-binding partner (DP1-3) (Wu et al. 1995). Regulation of E2F transcriptional function is controlled at several levels, including expression of the E2F compo-

${ }^{3}$ Corresponding author.

E-MAIL vousden@ncifcrf.gov; FAX (301) 846-1666. nents (Kaelin Jr. et al. 1992; Slansky et al. 1993), phosphorylation of each binding partner (Krek et al. 1994) and interaction with one of the $\mathrm{pRB}$ family of pocket proteins that includes pRB, p107, and p130 (Weinberg 1995). Although this interaction does not inhibit the DNA-binding activity of E2F, when complexed with $\mathrm{pRB}, \mathrm{E} 2 \mathrm{~F}$ is converted from an activator of transcription to a potent transcriptional repressor (Adams and Kaelin 1995). Indeed, although some of the genes necessary for cell cycle progression are transcriptionally activated by E2F, several genes have been shown to be actively transcriptionally repressed through E2F-binding sites within their promoters, with activation of expression being related to loss of repression, rather than positive transcriptional actiration (Lam and Waston 1993; Johnson et al. 1994; Neuman et al 1994). During cell cycle progression, transcriptionally repressive complexes of $\mathrm{E} 2 \mathrm{~F}$ with $\mathrm{pRB}$ seen during $G_{1}$ are lost as a result of phosphorylation of $p R B$ by a member of the $G_{1}$ cyclin dependent kinases, which leads to the dissociation of the pRB/E2F complex (Weinberg 1995). Loss of transcriptional repression and the generation of free E2F with positive transcriptional activity results in the expression of genes necessary for DNA synthesis and progression into $S$ phase. Later, at the end of $S$ phase, cyclin A-associated kinase directly complexes with the E2F component of the transcription factor that is subsequently inactivated following phosphorylation of the DP subunit (Krek et al. 1995). This 
inactivation of E2F function allows exit from S-phase and continued cell cycle progression.

Despite the clear importance in allowing cell cycle progression, recent studies have shown that one of the components of the E2F family, E2F-1, also shows apoptotic functions under conditions of deregulated expression. In mice, interference with the regulation of E2F activity provided by the pRB protein family, either by deletion of the $\mathrm{pRB}$ gene or expression of viral proteins that inhibit $\mathrm{pRB}$ function, results in unregulated cell proliferation and apoptosis (Pan and Griep 1994; Almasan et al. 1995). Similarly, ectopic expression of E2F-1 in quiescent fibroblasts leads to entry into DNA synthesis and apoptosis (Qin et al. 1994; Shan and Lee 1994; Kowalik et al. 1995). Several proteins that play a role in regulating cell cycle progression also have apoptotic activities and it has been suggested that the apoptotic signal induced by such proteins is a direct consequence of their growth control functions. In such a conflict of signals model, simultaneous expression of growth promoting and growth arrest signals results in the activation of the cell death pathway. Cooperation between E2F activity and the tumor suppressor protein p53 (Morgenbesser et al. 1994; Wu and Levine 1994) has lead to the suggestion that cell death may be mediated by a conflict of growth stimulatory signals activated by E2F and the growth inhibitory functions of $\mathrm{p} 53$. This model implies that the apoptotic activity of deregulated E2F is a consequence of unscheduled cell cycle progression induced by E2F itself and is supported by data from several studies. In particular, analysis of E2F-1-induced apoptosis in mouse fibroblasts has clearly shown that loss of the normal regulation of E2F-1 function through inactivation by cyclin A at the end of $S$ phase provides an apoptotic stimulus (Krek et al. 1995). The observation that E2F-1-deficient mice show an impaired apoptotic function and increased incidence of cancer development (Field et al. 1996; Yamasaki et al. 1996), however, suggests that E2F-1 may also play a more direct role as a tumor suppressor, independent of E2F induced cell proliferation. It is also of interest to note that loss of E2F-1 function had no profound effect on the development of these mice, illustrating that the cell cycle progression function of E2F-1 is not essential during development.

Although p53 can augment E2F-1-induced apoptosis in several systems, previous studies had suggested that the growth inhibitory effects of E2F-1 are not entirely dependent on p53 (Melillo et al. 1994; Qin et al. 1994, 1995; Berry et al. 1996) and pRB has been shown to protect p53 null human cells from apoptosis in an E2F-1 bindingdependent manner (Haas-Kogan et al. 1995). A p53-independent apoptotic activity of E2F is strongly supported by a recent study in mice, showing that deletion of $R b$ (and therefore deregulation of E2F) results in apoptosis in both p53 positive and negative embryos (Macleod et al. 1996). In this study we have shown directly that E2F-1 has p53-independent apoptotic activities in human cells and that E2F-1 apoptotic function is not dependent on transcriptional activation or the ability to induce cell cycle progression.

\section{Results \\ Contribution of p53 to E2F-1-induced apoptosis}

E2F-1 and p53 have been shown to cooperate in the induction of apoptosis. Expression of p53 leads to both a cell cycle arrest and apoptosis in p53 null human tumor cell lines such as Saos-2 or H1299, although the apoptotic activity of p53 itself can be dissociated from cell cycle arrest (Rowan et al. 1996). To examine the contribution of p53 induced cell cycle arrest to E2F-1-mediated apoptosis, we analyzed Saos-2 cells transfected with E2F-1 and p53 (Fig. 1A) using a flow cytometric assay that measures apoptotic rate at the time of harvesting rather than cumulative apoptosis (Rowan et al. 1996). Although high levels of p53 expression can induce substantial apoptosis in these cells, we chose to examine the effects of lower levels of $\mathrm{p} 53$ with a plasmid in which $\mathrm{p} 53$ expression is under the control of the MoLTR, a relatively weak promoter in these cells. Under these conditions, p53 induces only a low rate of apoptosis, allowing the retention of sufficient sensitivity to detect any increase in apototic rate. As described in several other systems, an enhanced apoptotic rate was seen following coexpression of E2F-1 and p53 (Fig. 1A). Protein analysis by Western blotting showed expression of the transfected p53 and E2F-1 in these cells (Fig. 1B). Very low levels of endogenous E2F-1 were also seen in the absence of transfected protein, although these endogenous levels were not always within the limits of detection in subsequent analyses.

In addition to enhancing the p53-dependent apoptosis, expression of E2F-1 alone, in the absence of p53, also showed an apoptotic response (Fig. 1A). A closer analysis of cells at 24 and $72 \mathrm{hr}$ post-transfection revealed clearly elevated apoptotic rates in E2F-1 expressing cells, despite the absence of p53 (Fig. 1C), which were significantly enhanced by the absence of serum. The ability of E2F-1 to induce p53-independent apoptosis was confirmed in a second p53 null human tumor line, H1299 (data not shown). To further confirm that E2F-1 expression can lead to apoptosis in the absence of p53, we analyzed transfected Saos- 2 cells by staining directly for E2F-1 expression (Fig. 1D). Cells sorted on the basis of E2F-1 overexpression again showed an enhanced apoptotic rate compared with the background, untransfected population. We were concerned that the p53-independent apoptotic function of E2F-1 might be a consequence of excessive overexpression of the exogenous protein, both on the basis of the Western blot analysis (Fig. 1B) and the flow cytometric analysis of transfected cells stained directly with an anti-E2F-1 antibody (Fig. 1D), which revealed cells with a range of fluorescence up to 3 logs higher than background. However, gating out very high expressing cells and analyzing the cell cycle profile of the population of cells with fluorescence $<10$-fold higher than background generated essentially identical data to those described above (data not shown), indicating that p53-independent E2F-1-induced apoptosis is not dependent on extremely high levels of overexpression. Because our results indicated that E2F-1 has apoptotic activity in 


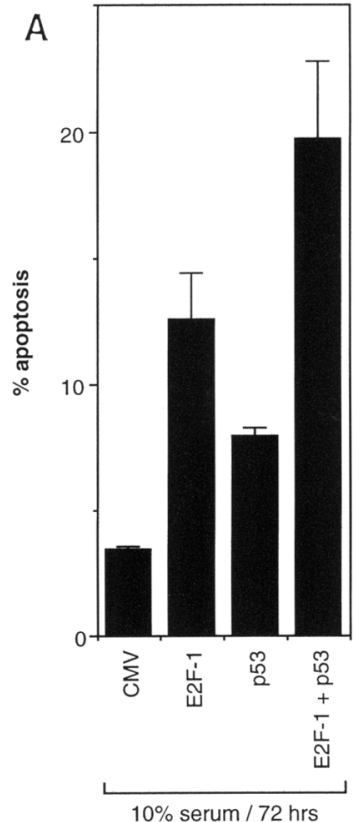

D

CMV
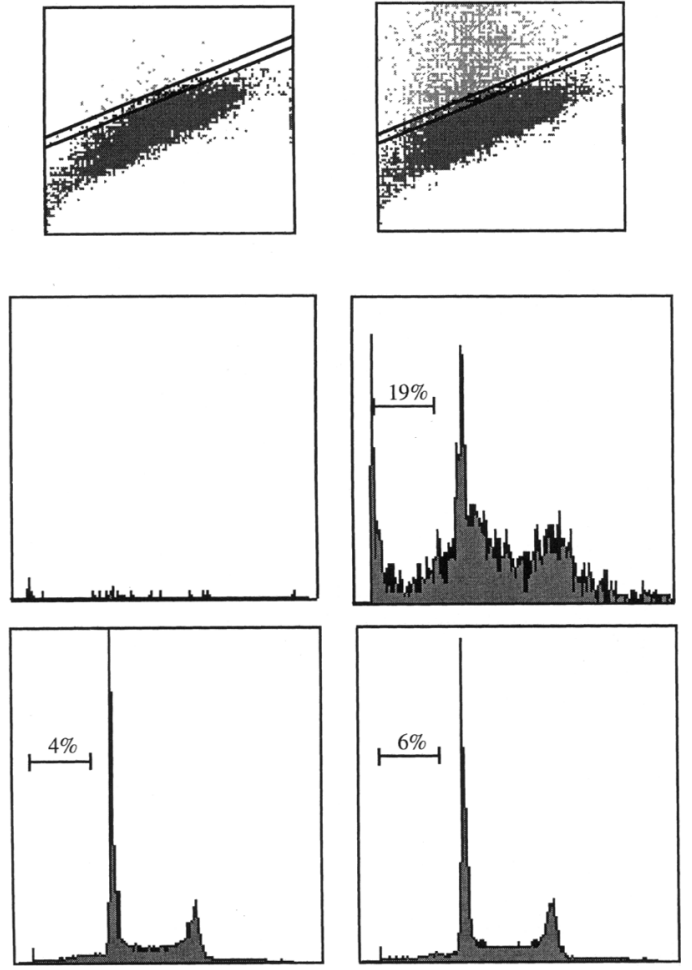

E2F-1

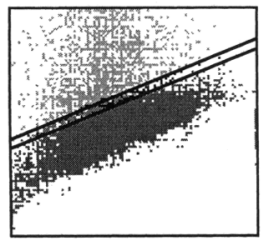



Figure 1. E2F-1 induces p53 independent apoptosis in Saos-2 cells. Cells were transiently transfected with $1 \mu \mathrm{g}$ of p53 and/or E2F-1 expression plasmids as indicated and analyzed by FACS. The increase in apoptosis (as determined by cells with a sub-G $_{1}$ DNA content) above that is seen in the untransfected population (typically $2 \%-6 \%$ with serum, $5 \%-15 \%$ without serum) is shown. (A) Apoptosis $72 \mathrm{hr}$ following transfection with vector (CMV), p53, or E2F-1 expression plasmids as indicated. $(B)$ Western blot analysis of cells shown in $A$, demonstrating expression of E2F-1 and p53. (C) Apoptosis 24 and $72 \mathrm{hr}$ following transfection with vector (CMV) or E2F-1 expression plasmid, in the presence or absence of serum. $(D)$ FACS analysis of cells stained directly for E2F-1 expression $72 \mathrm{hr}$ following transfection. The gates are established with vectoronly transfected cells (CMV) (top), allowing separate analysis of E2F-1 overexpressing cells (middle) and the untransfected population (bottom). Percentage of cells with a sub-G ${ }_{1}$ DNA content is indicated. the absence of $\mathrm{p} 53$, we continued to analyze this function in p53 null cell lines.

Activation of cell cycle progression is not necessary for E2F-1 induced apoptosis

Although p53 is clearly not necessary for the E2F-1-in- duced apoptotic signal, the well-established cooperation between p53 and E2F-1 may reflect the conflict between E2F-1 proliferative and p53 cell cycle arrest activities. It is also possible, therefore, that elevated apoptosis seen following ectopic expression of E2F-1 in serum deprived Saos- 2 cells is the result of the conflicting signals generated by the E2F proliferative signal in the absence of 
Phillips et al.

exogenous growth factors. In an attempt to understand the mechanism of E2F-1-mediated p53-independent apoptosis, we utilized a number of previously characterized E2F-1 mutants (Helin and Harlow 1994) (Fig. 2A). Because the ability of E2F-1 to drive cells through cycle has been shown to require an intact DNA-binding and transactivation domain, we sought to analyze the importance of E2F-1 transactivation activity to the apoptotic function. To do this, we tested two E2F-1 mutants in which the transactivation domain was deleted [E2F-1/1$374)$ and E2F-1(1-284)] and one point mutant [E2F$1(132 E)]$ that fails to bind DNA. As shown in other cell systems (Lukas et al. 1996), all the mutants were transcriptionally inactive as measured by activation of an E2F dependent promoter and all showed dominant-negative ability to inhibit transcriptional activation by the wild-type protein (Fig. 2B and data not shown). The E2F$1(1-374)$ and E2F-1(1-284) mutants, which retain the ability to bind DNA, are likely to inhibit wild-type E2F-1 activity by competitively binding to E2F sites. The nonDNA-binding mutant E2F-1(132E) retains the ability to interact with the DP-binding partner and competes with wild-type E2F-1 for the formation of an active dimeric complex. Western blot analysis of cells transfected with $1 \mu \mathrm{g}$ of each E2F-1 expression plasmid showed similar levels of each E2F-1 protein in the transfected cells during the course of these transient assays. However, the two carboxy-terminal deletion mutants E2F-1 (1-374) and E2F-1 (1-284) were consistently expressed at slightly higher levels than the wild-type E2F-1 or E2F-1(E132) proteins. These observations are consistent with the recent observation that sequences within the carboxyl terminus of E2F-1 confer protein instability, and deletion of this region stabilizes the protein (Hoffmann et al. 1996).

To analyze the correlation between promotion of entry into DNA synthesis and apoptosis induced by E2F-1, cell cycle profiles of Saos- 2 cells transiently transfected with each E2F-1 mutant were examined in the absence of serum, conditions under which maximum apoptosis was induced by wild-type E2F-1 (Fig. 1C). Although these cells do not become quiescent in the absence of serum, a retardation of cell cycle progression can be seen with
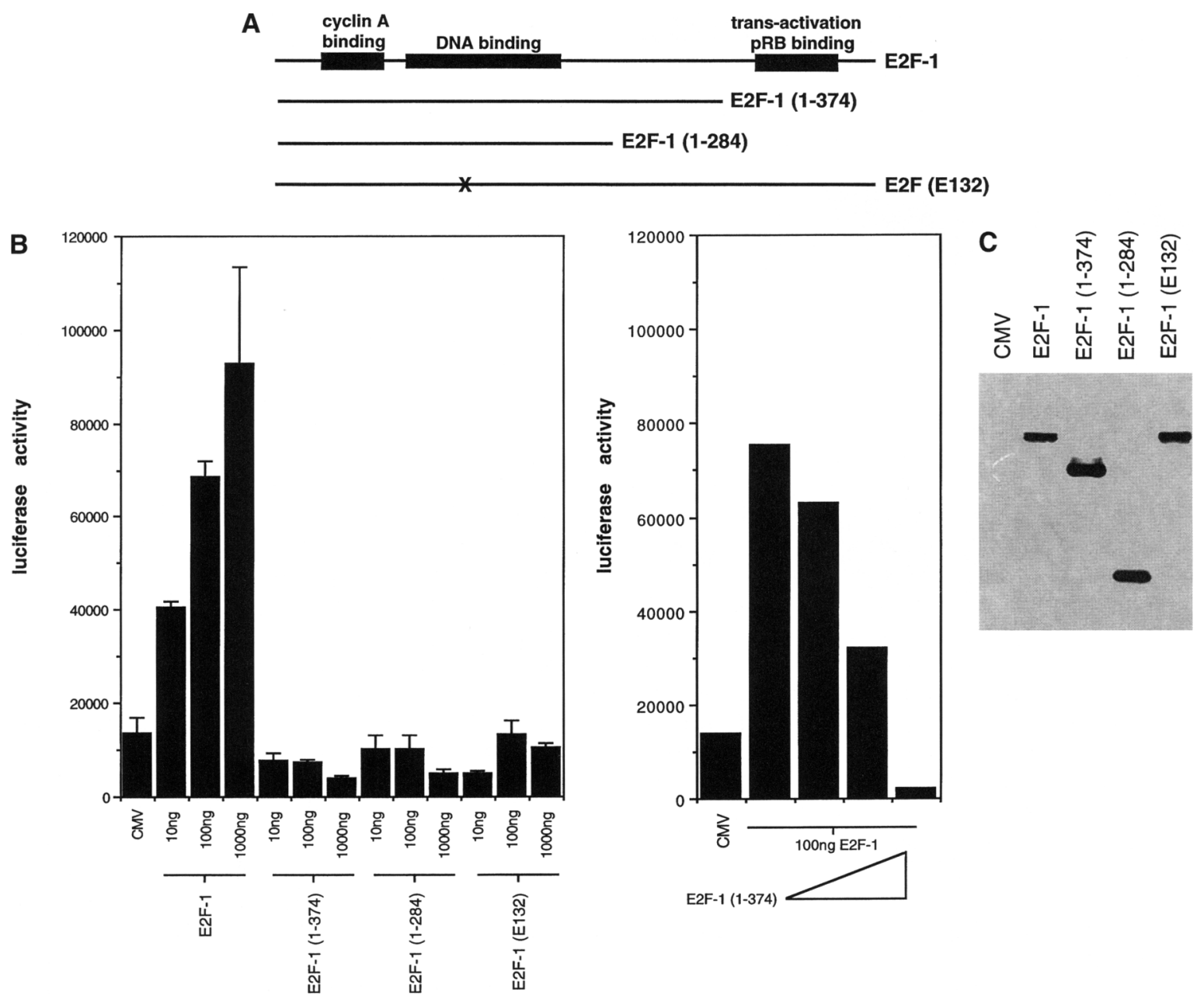

Figure 2. Characterization of E2F-1 mutants in Saos-2 cells. (A) Functional domains of E2F-1 and mutants used in this study. (B) Activation of an E2F dependent reporter gene by wild-type E2F-1, but not mutant E2F-1, and repression of wild-type E2F-1 transcriptional activity by E2F-1(1-374) in Saos-2 cells harvested $24 \mathrm{hr}$ post-transfection. (C) Expression of E2F-1 and mutants in Saos-2 cells transiently transfected with $1 \mu \mathrm{g}$ of each E2F-1 expression plasmid as in $B$. 
some accumulation of cells in $\mathrm{G}_{1}$. As shown previously, whereas expression of wild-type E2F-1 accelerates the rate at which cells enter $S$ phase, each of the transcriptionally inactive mutants further slowed progression through the cell cycle leading to an increased accumulation of cells in $G_{1}$ (Lukas et al. 1996) (Fig. 3A). This impediment to cell cycle progression is likely to be a consequence of interference with the endogenous E2F function in these cells, either by competing for DNAbinding sites or by titration of the DP-binding partners. Surprisingly, however, both transactivation defective E2F-1 mutants [E2F-1(1-374) and E2F-1(1-284)] induced apoptosis at least as efficiently as wild-type E2F-1, although the non-DNA-binding E2F-1(E132) mutant failed to induce apoptosis (Fig. 3A). Comparable levels of expression of each E2F-1 protein was seen under conditions of serum starvation (Fig. 3B). In light of the unexpected apoptotic activity of the two transcriptionally inactive E2F-1 mutants, we repeated the analysis in cells maintained in the presence of serum, conditions that had markedly reduced the apoptotic potential of wild-type E2F-1 (Fig. 1C). The ability of E2F-1 to promote cell cycle progression was also evident in the presence of serum and as seen in serum-deprived cells, all three transcriptionally inactive mutants failed to accelerate cell cycle progression and induced some degree of cell cycle arrest in the presence of serum (Fig. 3C,D). Although addition of serum significantly reduced wild-type E2F-1 mediated apoptosis, serum had only a modest effect on the cell death induced by E2F-1(1-374) or E2F-1(1-284). Because the transactivation deficient mutants were slightly more stably expressed than the wild-type protein (Fig. 2C), we analyzed the effect of transfecting $5 \mu \mathrm{g}$ of the wild-type E2F-1 expression plasmid into cells grown in the presence of serum. Under these conditions, wild-type E2F-1 expression was increased to levels equivalent to that seen with the two mutants, but apoptosis did not increase to match the levels seen with E2F-1(1-374) or E2F1(1-284) (data not shown). This suggests that the differences in apoptotic potential of wild-type E2F-1 and the trans-activation mutants is not simply a reflection of differences in levels of protein expression, although the possibility remains that we are underestimating apoptosis induced by wild-type E2F-1 by including cells dying from $G_{2}$ within the S-phase population. This is less likely to occur in cells expressing the mutants because they are predominantly in $\mathrm{G}_{1}$. The DNA-binding defective E2F-1(E132) mutant showed no apoptotic activity in any assay. The consistent failure of this mutant to induce apoptosis in either the presence or absence of serum indicated that the apoptotic function of the other mutants is also not simply a reflection of the titration of an E2F-1-binding protein such as DP1 or cyclin A, as a result of overexpression. Essentially identical data were obtained in H1299 cells (data not shown).

\section{Cell death induced by E2F-1 is apoptosis}

In view of the unexpected apoptotic function of the E2F mutants, we sought to confirm that the cell death seen was a result of apoptosis. A number of lines of evidence strongly indicate that this is the case. Coexpression of the apoptosis inhibitor Bcl-2 resulted in the inhibition of cell death induced by both wild-type E2F-1 and the E2F1(1-374) mutant (Fig. 4A). Western blot analysis of these cells showed that the protection afforded by Bcl-2 expression was not the result of a reduction in E2F-1 protein expression in the cotransfected cells (Fig. 4B). Examination of transfected cells morphology by DAPI staining revealed nuclear blebbing and apoptotic bodies in a significant number of E2F-1(1-374) expressing cells in the presence of serum (data not shown). A TUNEL assay, which measures the presence of nicked DNA, an early event in apoptosis, showed that a significantly higher proportion of cells expressing E2F-1(1-374) were TUNEL positive, compared to either E2F-1 or E2F-1(E132) expressing cells (Fig. 4C). These results show that the cell death generated by ectopic expression of E2F-1(1-374) shows morphological, biochemical, and biological features of apoptosis.

Cyclin $A$ binding is not necessary for p53-independent E2F-1-induced apoptosis.

Previous studies have shown that loss of negative regulation of E2F DNA-binding activity by cyclin A associated kinase results in the inability of cells to leave Sphase and eventual apoptosis (Krek et al. 1995). Because this apoptotic function depends on the ability of E2F-1 to drive DNA synthesis, it is dependent on the presence of the transcriptional activation domain and the transactivation-deficient E2F-1 mutants show loss of this activity. The E2F-1 apoptotic activity described here is clearly distinct from the previously described function, and we were interested to determine whether the interaction of cyclin A with E2F-1 could also modulate the present apoptotic function. Analysis of E2F-1 mutants deleted of the cyclin A-binding site (E2F-1 $\Delta 24)$ (Krek et al. 1995) (Fig. 2A), which showed enhanced apoptotic activity at the end of S-phase, behaved indistinguishably from the wild-type protein in the present assay (Fig. 5A), indicating that loss of regulation of E2F-1 by cyclin A does not contribute to the apoptotic function being measured here. Conversely, loss of the cyclin A-binding domain did not significantly reduce the apoptotic activity of the transactivation domain deleted E2F-1 mutant [E2F-1 $\Delta 24(1-374)]$, showing that cyclin A binding is not necessary for this function. Protein analysis showed similar expression levels for all the E2F-1 proteins in the transfected cells (Fig. 5B), although slightly enhanced expression of the mutants lacking the carboxy-terminal destabilization domain was seen again.

The expression of several genes with roles in regulating entry and progress through DNA synthesis has been shown to be regulated by E2F, including B-myb (Lam and Waston 1993), cyclin E (Ohtani et al. 1995), and cyclin A (Schulze et al. 1995). The apoptotic activities of the E2F-1 mutants indicated that transcriptional activation is not necessary to induce cell death, but the loss of function displayed by the DNA-binding mutant sug- 
Phillips et al.

gested that relief of transcriptional repression through E2F-binding sites may play a role. Transactivation domain deletion mutants of E2F-1 have been shown to retain the ability to block E2F-1-mediated transcriptional repression (Qin et al. 1995). Transcriptional repression through E2F-binding sites has been described for several cell promoters, including the B-myb promoter (Lam and Waston 1993) and the cyclin A promoter, which is repressed during the early stages of the cell cycle through a variant E2F-binding site (Schulze et al. 1995). Although any of these genes may be downstream mediators of the E2F-1 apoptotic response seen here, preliminary analysis of the consequence of ectopic cyclin A expression in these cells did not reveal any apoptotic activity, although accelerated cell cycle progression could be detected (data not shown).

\section{Discussion}

Our analysis of E2F-1 apoptotic function has shown that p53 is not essential for an E2F-1-induced apoptotic signal, and that the apoptotic activity of E2F-1 can be dissociated from the ability to accelerate cell cycle progression. The contribution of p53 to E2F-1-mediated apoptosis has been well established and we have also observed elevated apoptosis in cells transfected with p53 and E2F1. The evidence is compelling that in some cell types in vivo, p53 plays an important role mediating this function of E2F-1. Nevertheless, the p53-independent apoptotic activity of E2F-1 described here is consistent with several previous studies that have documented a growth inhibitory effect of E2F-1 in similar p53 negative cells
(Melillo et al. 1994; Qin et al. 1994; Berry et al. 1996). Furthermore, recent analyses of $R b-/-$ mice in $p 53-/-$ or $+/+$ backgrounds have clearly demonstrated p53 independent apoptosis mediated by loss of pRB in some tissues (Macleod et al. 1996). Our experiments suggest that the p53-independent apoptosis seen in this mouse model is mediated by E2F-1, and that this apoptotic activity of E2F-1 is physiologically important. The elimination of p53 from our experiments has also allowed a simpler dissection of the mechanisms by which E2F-1 activates cell death in the absence of a p53-mediated cell cycle arrest.

Although conflicting signals for cell cycle arrest and cell cycle progression may activate an apoptotic response, we show here that E2F-1 can exhibit separable cell cycle progression and apoptotic activities. Cell cycle progression and apoptotic functions have also been dissociated for the transcription factor c-Myc, where c-Myc induced apoptosis can be separated from c-Myc induced proliferation (Harrington et al. 1994; Packham et al. 1996; Rudolph et al. 1996). Similarly, p53 induced cell cycle arrest is separable from p53 apoptotic activity (Rowan et al. 1996), and although p53 can cooperate with E2F-1 in the induction of apoptosis, we have found that activation of a cell cycle arrest by direct expression of the p53 target p21 WAF1/CIP1 does not enhance E2F-1-induced apoptosis (A.C. Phillips, unpubl.). Although the apoptotic function of E2F-1 does not appear to depend on E2F-1-dependent transcriptional activation, the inactivity of the E2F-1(E132) mutant indicates the importance of DNA binding. The presence of E2F-binding sites within some promoters has been shown to be respon-
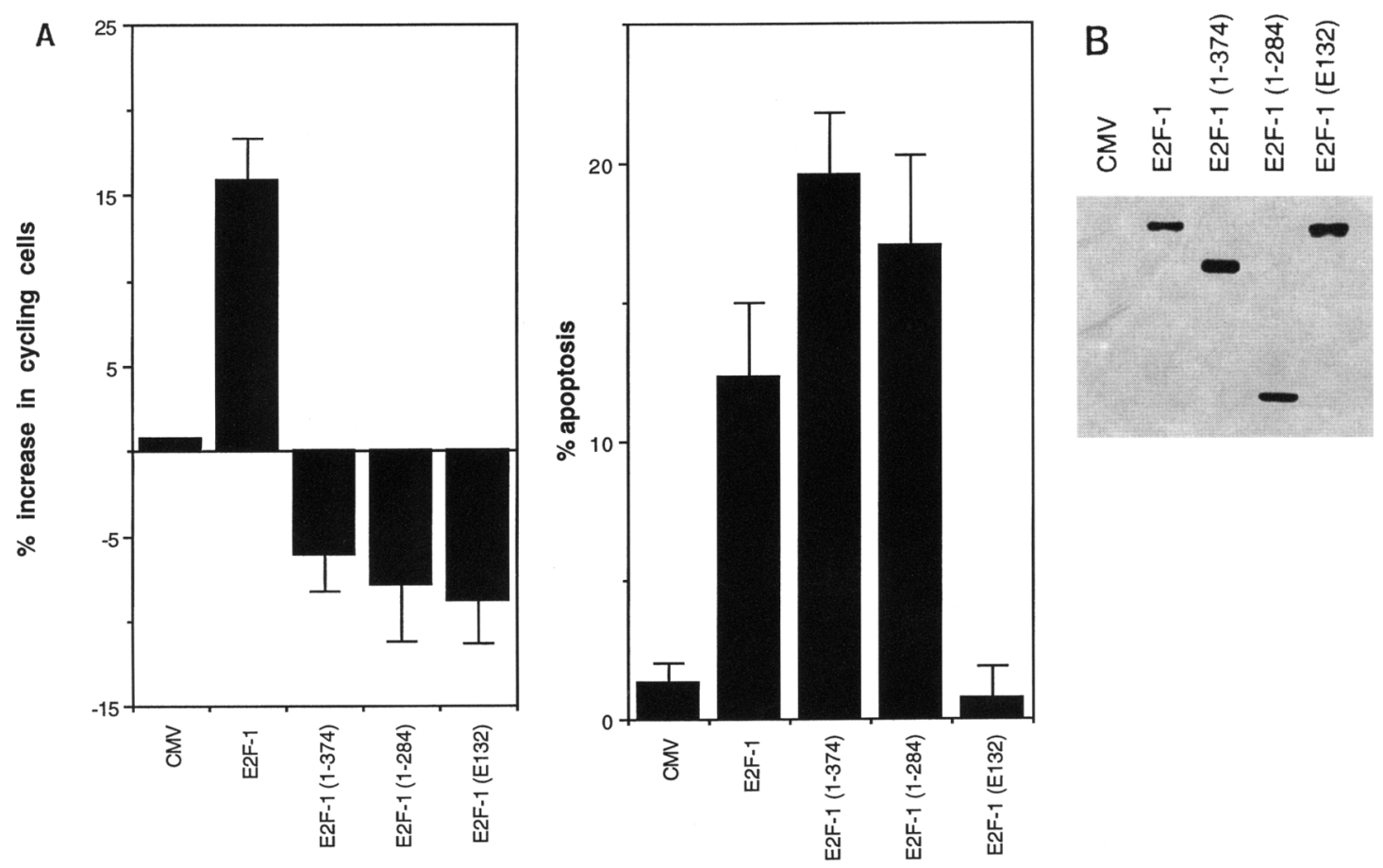

Figure 3. (See facing page for $C, D$, and legend.) 
E2F-1 apoptotic function

C
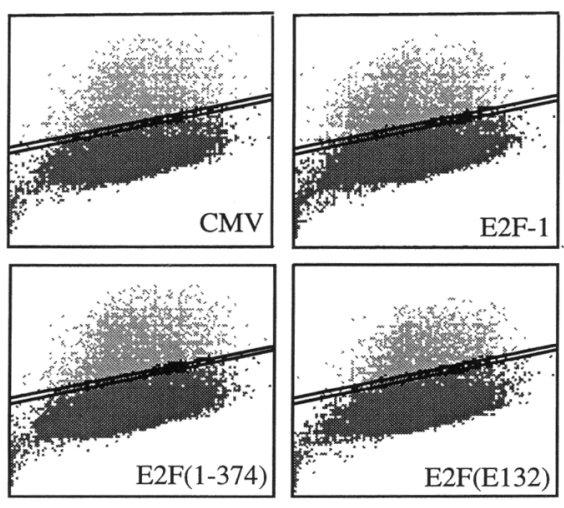
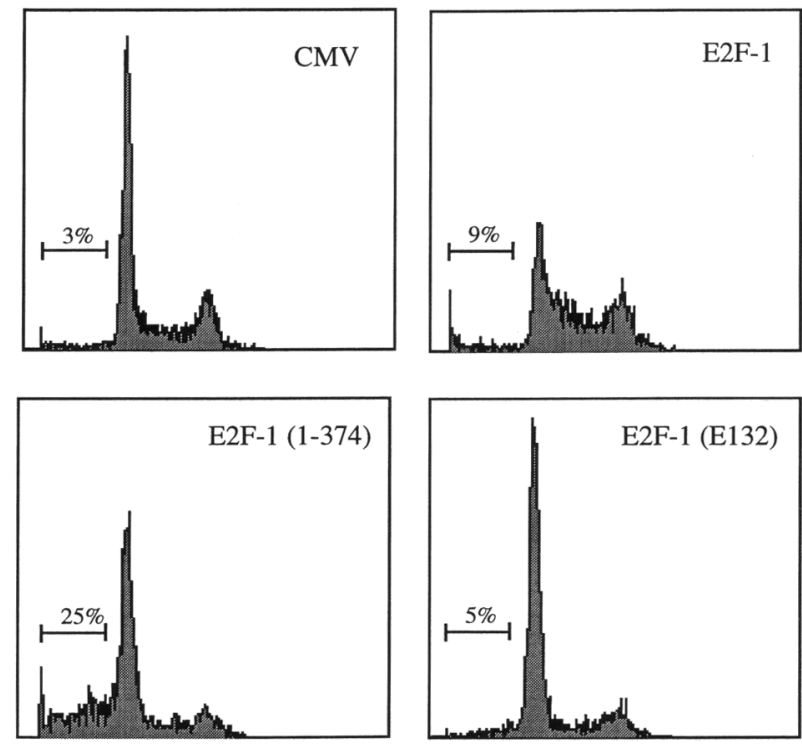
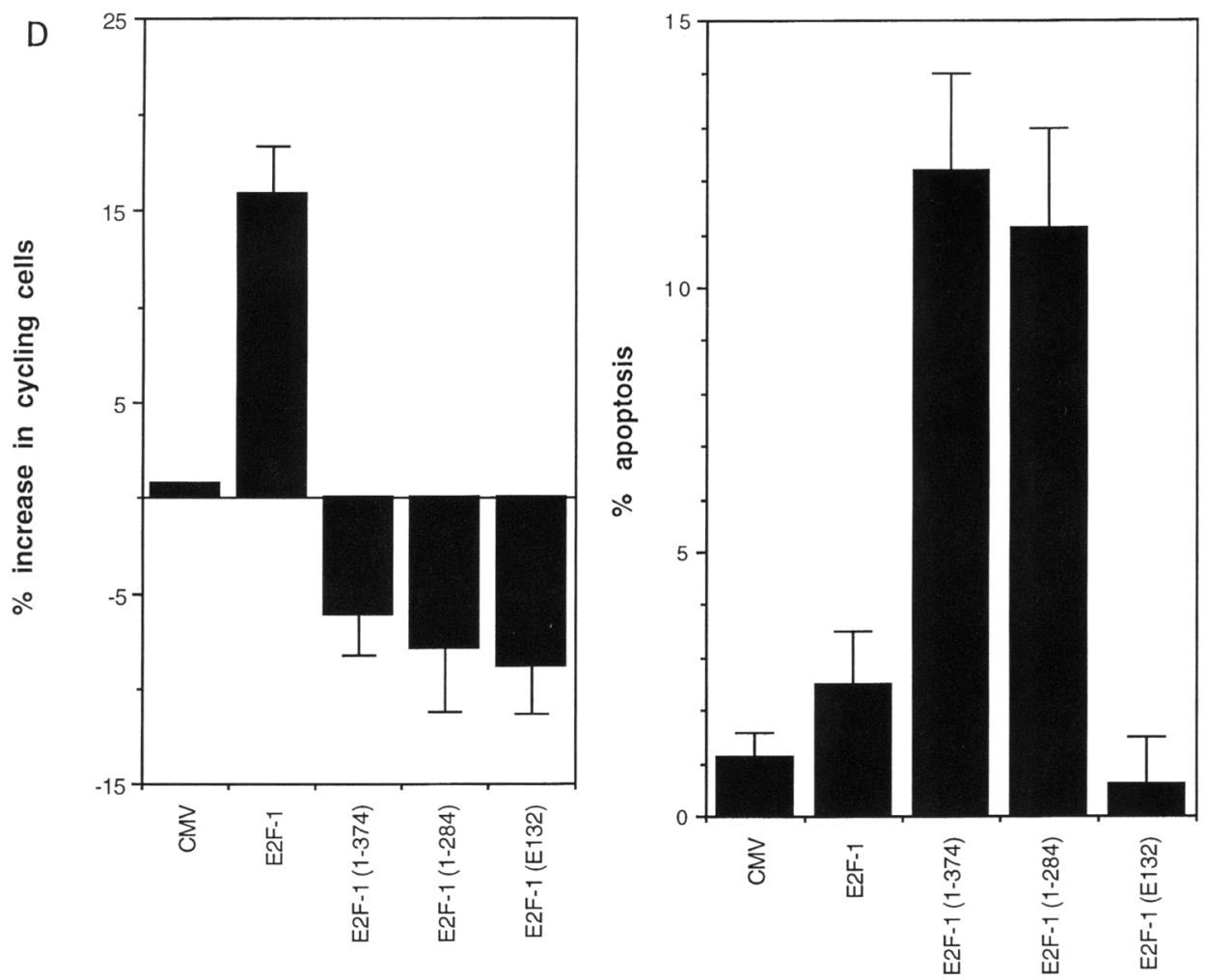

Figure 3. Apoptotic activity of E2F mutants lacking a transactivation domain. Cells were transiently transfected with $1 \mu \mathrm{g}$ of each E2F-1 expression plasmid as indicated and analyzed by FACS. Changes in the proportion of transfected cells in cycle (i.e., in S, $\mathrm{G}_{2}$, or M) and undergoing apoptosis (i.e., in the sub- $G_{1}$ ) relative to untransfected cells are shown. (A) Cell-cycle progression and apoptotic activities of wild-type E2F-1 and the E2F-1 mutants in Saos-2 cells in the absence of serum. Cells were harvested 24 hr posttransfection. (B) Expression of E2F-1 and mutants in Saos-2 cells analyzed in $A$, in the absence of serum. $(C)$ Representative FACS profiles of Saos-2 cells transfected with vector, wild-type E2F-1, E2F-1(1-374), and E2F-1(E132) in the presence of serum, 48 hr post-transfection. These cells were cotransfected with the CD20 expression plasmid and sorted on the basis of CD20 expression (1eft). Cell-cycle analysis of the CD20-expressing cells is shown (right). (D) Cell-cycle progression and apoptotic activities of wild-type E2F-1 and the E2F-1 mutants in Saos-2 cells in the presence of serum. Cells were harvested $24 \mathrm{hr}$ post-transfection. 
A
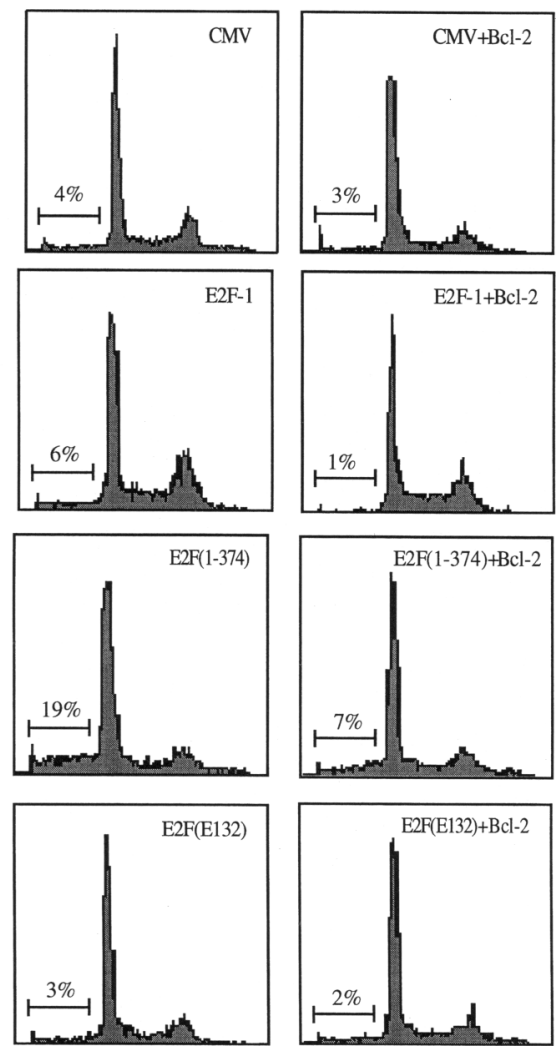

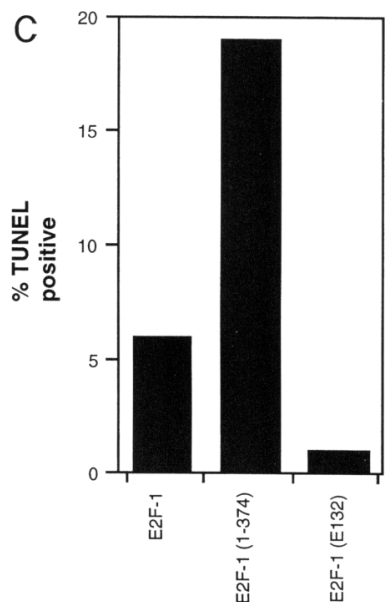

Figure 4. Cell death induced by E2F-1 apoptosis. (A) Cell death mediated by E2F-1 in the presence of serum is inhibited by Bcl-2. Cell-cycle profiles of Saos-2 cells are shown transiently transfected in the presence of serum with the indicated expression plasmids. Cells were harvested $24 \mathrm{hr}$ post-transfection and percentage of cells with a sub- $G_{1}$ DNA content is indicated. This experiment was repeated three times with similar results. $(B)$ Expression of E2F-1 proteins and Bcl-2 in transiently transfected Saos-2 cells; $(C)$ Expression of E2F-1 results in an increase of TUNEL-positive cells. Saos-2 cells were transiently transfected in the presence of serum with E2F-1 or E2F mutant expression plasmids and 200 E2F-1 positive cells for each sample were scored for TUNEL activity. sible for repression of transcription at certain stages of the cell cycle, mediated by binding of E2F/pocket protein complexes (Lam et al. 1994; Sellers et al. 1995; Weintraub et al. 1992, 1995). The importance of DNAbinding activity to the E2F-1 apoptotic function described in this study suggests that this apoptotic activity may reflect the alleviation of E2F-mediated transcriptional repression, rather than the activation of E2F responsive genes. In support of this suggestion, the accompanying paper by Hsieh et al. shows that $\mathrm{pRB}$ can inhibit the apoptotic function of wild-type E2F-1, but not the E2F-1(1-374) mutant that fails to bind pRB. It should be pointed out, however, that most of our studies were carried out in Saos-2 cells, which fail to express wild-type $\mathrm{pRB}$. Although this tumor cell line may have acquired additional alterations that protect it from the apoptotic activity of the endogenous E2F-1, the observation that enhanced expression of E2F-1 can induce apoptosis in these cells suggests that, under some circumstances, repression of the apoptotic target genes may also be carried out by E2F complexes containing other pocket proteins such as p107 and p130.

The expression of several cellular genes has been shown to be repressed through E2F-binding sites and any of these may be apoptotic targets for E2F-1 transcriptional activation. We have noted that expression of cyclin A can show an ability to promote cell cycle progression similar to that seen for E2F-1 without inducing apoptosis, further supporting the model in which the apoptotic function of E2F-1 is not simply a consequence of activation of cell cycle progression. Nevertheless, previous studies with p53 have shown that protein levels can be of critical importance in determining whether a cell undergoes cell cycle perturbations or apoptosis (Chen et al. 1996) and we cannot rule out an apoptotic activity of cyclin A. It is possible that several E2F-1regulated genes contribute to the apoptotic response, and we are generating cell lines with inducible E2F-1 in which to identify the critical target genes. It is of interest, however, that activation of cyclin-dependent kinases and induction of cyclin A by c-Myc has been shown to be independent of the Myc-induced apoptotic signal (Rudolph et al. 1996).

Failure to exit $S$ phase, through phosphorylation and inactivation of E2F activity by cyclin A dependent kinase, has been shown to lead to apoptosis in rodent fibroblasts, and this activity requires the transactivation domain in E2F-1 (Krek et al. 1995). In contrast, the apoptotic activity of E2F-1 described in this study does not appear to depend on the ability of E2F-1 to induce Sphase progression and we would therefore like to propose that E2F-1 can generate an apoptotic signal at more than one stage of the cell cycle. The apoptotic function described here appears to be suppressed under conditions of normal cell growth, only becoming evident following a perturbation of normal cell cycle progression. In our model (Fig. 6), release of free E2F-1 from pocket protein interactions (either by ectopic overexpression or normal cell cycle progression/ simultaneously activates expression of genes necessary for DNA synthesis and dere- 



Figure 5. Contribution of the cyclin A-binding domain of E2F-1 to apoptosis in Saos-2 cells. (A) Saos-2 cells were transiently transfected with E2F-1 expression plasmids as indicated in the presence of serum and analyzed by FACS. Cells were harvested $24 \mathrm{hr}$ post-transfection. Changes in the proportion of transfected cells in cycle (i.e., in $S, G_{2}$, or $M$ ) and undergoing apoptosis (i.e., in the sub-G $\mathrm{G}_{1}$ population) compared to untransfected cells are shown. (B) Western blot analysis of E2F-1 expression in Saos-2 cells shown in $A$.

presses expression of the apoptotic genes. Under conditions that allow progress into $S$ phase, the apoptotic signal is suppressed. However, the apoptotic activity is revealed in the absence of normal cell cycle progression, either following serum starvation or expression of transactivation domain E2F-1 mutants, which prevent activation of the appropriate S-phase-specific genes while retaining the ability to derepress expression of the apoptotic targets (Qin et al. 1995). The DNA-binding mutant fails to induce apoptosis because it is defective for both activation of S-phase genes and derepression of apoptotic genes. According to this model, ectopic expression of $\mathrm{pRB}$ would result in maintenance of transcriptional repression of the apoptotic targets, although the apoptotic activity of E2F-1 in pRB-deficient Saos-2 cells clearly complicates the interpretation of the results. Nevertheless, our model would predict a function for E2F-1 in monitoring conditions of cell cycle progression, and activation of an apoptotic function in response to perturbations of normal cell proliferation is consistent with the tumor suppressor activity of E2F-1.

\section{Materials and methods \\ Constructs and antibodies}

The E2F-1 expression constructs have been described previously (Herlin and Harlow 1994; Krek et al. 1995; Rowan et al. 1996), as have the E2F-1 reporter construct (Zamanian and La Thangue 1992) and the pCMVCD20 expression plasmid (Zhu et al. 1993). p53 expression was driven from the MoLTR in the construct pJ4 2 p53 (Crook et al. 1994b), human Bcl-2 cDNA was cloned into pcDNA3 (Invitrogen) for expression from the CMV promoter. Where appropriate, transfection efficiency was moni-

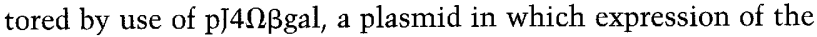
$\beta$-galactosidase gene is under the control of the MoLTR /Crook et al. 1994a). For detection of E2F-1, the monoclonal antibody KH20 was used (Helin et al. 1993). CD20 expression was detected by use of FITC conjugated anti-CD20 monoclonal antibody (Becton Dickinson), p53 expression was detected by use of the monoclonal antibody DO-1 (Vojtesek et al. 1992) and Bcl-2 expression was detected with a polyclonal rabbit antiserum

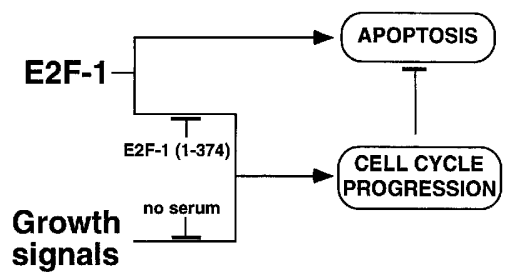

Figure 6. Model of E2F-1 function. E2F-1 activates transcription of genes necessary for cell-cycle progression and de-represses expression of apoptotic genes. In the presence of growth signals (serum), normal cell-cycle progression suppresses the apoptotic signal. Failure of normal cell-cycle progression, either through loss of growth signals or inability to activate E2F-1 responsive cell-cycle progression genes, results in the manifestation of the apoptotic response. 
Phillips et al.

raised against a $\mathrm{Bcl}-2$ peptide corresponding to amino acids $41-54$.

\section{Cell culture and transfection}

Saos- 2 and H1299 cells were maintained in Dulbecco's modified Eagle medium (DMEM) supplemented with $10 \%$ fetal calf serum. For transient transfection, $4 \times 10^{5}$ (for reporter gene assays) or $8 \times 10^{5}$ [for fluorescence-activated cell sorting (FACS) analysis] were seeded onto $10 \mathrm{~cm}$ dishes and transfected the following day, by use of calcium phosphate coprecipitation. Unless otherwise stated, all transfections were cotransfections of $1 \mu \mathrm{g}$ test plasmid with $0.5 \mu \mathrm{g}$ pCMVCD20, and the transient transfection efficiencies were within the range of $5-10 \%$. Cells were harvested after $24-72 \mathrm{hr}$ and prepared for flow cytometry or luciferase and $\beta$-galactosidase assays. In each case, one fifth of the sample was retained for protein analysis.

\section{Transcriptional activity and protein analysis}

Cell lysates were prepared $24 \mathrm{hr}$ after transfection and analyzed for luciferase and $\beta$-galactosidase activity as described previously (Lam and Waston 1993). For Western blotting, proteins in equal quantities of lysates were separated by $12.5 \%$ polyacrylamide gel electrophoresis and transferred overnight onto nitrocellulose filters.

\section{Flow cytometric analysis}

For flow cytometry, total populations of floating and adherent cells were harvested $24-72 \mathrm{hr}$ post-transfection, stained for CD20 expression, fixed in ethanol and stained for DNA content as described previously (Zhu et al. 1993). Cells stained directly for E2F-1 expression were fixed first and subsequently stained as described previously (Rowan et al. 1996). Flow cytometric analysis was carried out (FACScalibur, Becton Dickinson) and total cell populations were gated and analyzed as described previously (Rowan et al. 1996). Briefly, cells were measured for their FITC intensity (green channel) and propridium iodide fluorescence (red channel). Total populations were gated to remove doublets and very small debris. Background levels of FITC fluorescence were established by use of vector only transfected cells and CD20 or E2F-1 expressing cells were gated and analyzed for cell cycle distribution. TUNEL staining was carried out with the ApopTag Direct kit (Oncor) according to the manufacturer's instructions.

\section{Acknowledgments}

We thank Nick La Thangue for the E2F reporter construct, Ed Harlow for the CD20 expression construct, and William Krek for the E2F-1 $\Delta 24$ mutant. We are extremely grateful to Robert Ludwig for his expert assistance. This work was supported by the National Cancer Institute under contract with ABL, and by the Human Frontiers Science Program.

The publication costs of this article were defrayed in part by payment of page charges. This article must therefore be hereby marked "advertisement" in accordance with 18 USC section 1734 solely to indicate this fact.

\section{References}

Adams, P.D. and W.G. Kaelin. 1995. Transcriptional control by E2F. Semin. Cancer Biol. 6: 99-108.

Almasan, A., Y. Yin, R.E. Kelly, E.Y.-H.P. Lee, A. Bradley, W. Li,
J.R. Bertino, and G.M. Wahl. 1995. Deficiency of retinoblastoma protein leads to inappropriate S-phase entry, activation of E2F-responsive genes, and apoptosis. Proc. Natl. Acad. Sci. 92: 5436-5440.

Berry, D.E., Y. Lu, B. Schmidt, P.G. Fallon, C. O'Connell, S.-X. Hu, H.-J. Xu, and G. Blanck. 1996. Retinoblastoma protein inhibits IFN- $\gamma$ induced apoptosis. Oncogene 12: 1809-1819.

Chen, X., L.J. Ko, L. Jayaraman, and C. Prives. 1996. p53 levels, functional domains, and DNA damage determine the extent of the apoptotic response of tumor cells. Genes \& Dev. 10: 2438-2451.

Crook, T., C. Fisher, P.J. Masterson, and K.H. Vousden. 1994a. Modulation of transcriptional regulatory properties of $\mathrm{p} 53$ by HPV E6. Oncogene 9: 1225-1230.

Crook, T., N.J. Marston, E.A. Sara, and K.H. Vousden. 1994b. Transcriptional activation by p53 correlates with suppression of growth but not transformation. Cell 79: 817-827.

Field, S.J., F.-Y. Tsai, F. Kuo, A.M. Zubiaga, W.G. Kaelin, D.M. Livingston, S.H. Orkin, and M.E. Greenberg. 1996. E2F-1 functions in mice to promote apoptosis and suppress proliferation. Cell 85: 549-561.

Haas-Kogan, D.A., S.C. Kogan, D. Levi, P. Dazin, A. Tang, Y.K.T. Fung, and M.A. Israel. 1995. Inhibition of apoptosis by the retinoblastoma gene product. $E M B O /$. 14: 461-472.

Harrington, E.A., M.R. Bennett, A. Fanidi, and G.I. Evan. 1994. c-myc-induced apoptosis in fibroblasts is inhibited by specific cytokines. EMBO I. 13: 3286-3295.

Hartwell, L.H. and M.B. Kastan. 1994. Cell cycle control and cancer. Science 266: 1821-1828.

Helin, K. and E. Harlow. 1994. Heterodimerization of the transcription factors E2F-1 and DP-1 is required for binding to the adenovirus E4 (ORF6/7) protein. J. Virol. 68: 5027-5035.

Helin, K., C. Wu, A. Fattaey, J. Lees, B. Dynlacht, C. Ngwu, and E. Harlow. 1993. Heterodimerization of the transcription factors E2F-1 and DP-1 leads to cooperative transactivation. Genes \& Dev. 6: 177-185.

Hoffmann, F., F. Martelli, D.M. Livingston, and Z. Wang. 1996. The retinoblastoma gene product protects E2F-1 from degradation by the ubiquitin-proteasome pathway. Genes \& Dev. 10: 2949-2959.

Johnson, D.G., J.K. Schwarz, J.D. Cress, and J.R. Nevins. 1993. Expression of transcription factor E2F1 induces quiescent cells to enter S phase. Nature 365: 349-352.

Johnson, D.G., D. Ohtani, and J.R. Nevins. 1994. Autoregulatory control of E2F1 expression in response to positive and negative regulators of cell cycle progression. Genes \& Dev. 8: $1514-1525$.

Kaelin, W.G., Jr., W. Krek, W.R. Sellers, J.A. DeCaprio, F. Ajchenbaum, C.S. Fuchs, T. Chittenden, Y. Li, P.J. Farnham, M.A. Blanar, D.M. Livingston, and E.K. Flemington. 1992. Expression cloning of a cDNA encoding a retinoblastomabinding protein with E2F-like properties. Cell 70: 351-364.

Kowalik, T.F., J. Degregori, J.K. Schwarz, and J.R. Nevins. E2F1 overexpression in quiescent fibroblasts leads to induction of cellular DNA synthesis and apoptosis. I. Virol. 69: 24912500 .

Krek, W., M.E. Ewen, S. Shirodkar, Z. Arany, W.G. Kaelin, and D.M. Livingston. 1994. Negative regulation of the growthpromoting transcription factor E2F-1 by a stably bound cyclin A-dependent protein kinase. Cell 78: 161-172.

Krek, W., G. Xu, and D.M. Livingston. 1995. Cyclin A-kinase regulation of E2F-1 DNA binding function underlies suppression of an S-phase checkpoint. Cell 83: 1149-1158.

Lam, E.W.F. and R.J. Waston. 1993. An E2F-binding site mediates cell-cycle regulation repression of mouse B-myb transcription. EMBO I. 12: 2705-2713. 
Lam, E.W.F., J.D.H. Morris, R. Davies, T. Crook, R.J. Waston, and K.H. Vousden. 1994. HPV16 E7 oncoprotein deregulates b-myb expression - correlation with targeting of p107/E2F complexes. $E M B O$ I. 13: 871-878.

LaThangue, N.B. 1994. DRTF1/E2F - an expanding family of heterodimeric transcription factors implicated in cell-cycle control Trends Biochem. Sci. 19: 108-114.

Lukas, J., B.O. Petersen, K. Holm, J. Bartek, and K. Helin. 1996. Deregulated expression of E2F family members induces Sphase entry and overcomes p $16^{I N K 4 A}$-mediated growth suppression. Mol. Cell. Biol. 16: 1047-1057.

Macleod, K.F., Y. Hu, and T. Jacks. 1996. Loss of $R b$ activates both p53-dependent and independent cell death pathways in the developing mouse nervous system. EMBO I. 15: 61786188 .

Melillo, R.M., K. Helin, D.R. Lowy, and J.T. Schiller. 1994. Positive and negative regulation of cell proliferation by E2F-1: Influence of protein level and human papillomavirus oncoproteins. Mol. Cell. Biol. 14: 8241-8249.

Morgenbesser, S.D., B.O. Williams, T. Jacks, and R.A. Depinho. 1994. $p 53$-dependent apoptisis produced by $R b$-deficiency in the developing mouse lens. Nature 371: 72-74.

Neuman, E., E.K. Flemington, W.R. Sellers, and W.G. Kaelin. 1994. Transcription of the E2F-1 gene is rendered cell cycle dependent by E2F DNA-binding sites within its promoter. Mol. Cell. Biol. 14: 6607-6615.

Ohtani, K., J. DeGregori, and J.R. Nevins. 1995. Regulation of the cyclin E gene by transcription factor E2F1. Proc. Natl. Acad. Sci. 92: 12146-12150.

Packham, G., C. Porter, and J.L. Cleveland. 1996. c-Myc induces apoptosis and cell cycle progression by separable, yet overlapping, pathways. Oncogene 13: 461-469.

Pan, H.C. and A.E. Griep. 1994. Altered cell cycle regulation in the lens of HPV-16 E6 or E7 transgenic mice: Implications for tumor suppressor gene function in development. Genes \& Dev. 8: 1285-1299.

Qin, X.-Q., D.M. Livingston, W.G. Kaelin, and P.D. Adams. 1994. Deregulated transcription factor E2F-1 expression leads to S-phase entry and p53-mediated apoptosis. Proc. Natl. Acad. Sci. 91: 10918-10922.

Qin, X.-Q., D.M. Livingston, M. Ewen, W.R. Sellers, Z. Arany, and W.G. Kaelin. 1995. The transcription factor E2F-1 is a downstream target of RB action. Mol. Cell. Biol. 15: 742-755.

Rowan, S., R.L. Ludwig, Y. Haupt, S. Bates, X. Lu, M. Oren, and K.H. Vousden. 1996. Specific loss of apoptotic but not cell cycle arrest function in a human tumour derived p53 mutant. EMBO J. 15: 827-838.

Rudolph, B., R. Saffrich, J. Zwicker, B. Henglein, R. Müller, W. Ansorge, and M. Eilers. 1996. Activation of cyclin-dependent kinases by Myc mediates induction of cyclin A, but not apoptosis. EMBO T. 15: 3065-3076.

Schulze, A., K. Zerfass, D. Spitkovsky, S. Middendorp, J. Berges, K. Helin, P. Jansen-Dürr, and B. Henglein. 1995. Cell cycle regulation of the cyclin A gene promoter is mediated by a variant E2F site. Proc. Natl. Acad. Sci. 92: 11264-11268.

Sellers, W.R., J.W. Rodgers, and W.G. Kaelin. 1995. A potent transrepression domain in the retinoblastoma protein induces a cell cycle arrest when bound to E2F sites. Proc. Natl. Acad. Sci. 92: 11544-11548.

Shan, B. and W.H. Lee. 1994. Deregulated expression of E2F-1 induces S-phase entry and leads to apoptosis. Mol. Cell. Biol. 14: $8166-8173$.

Sherr, C.J. 1996. Cancer cell cycles. Science 274: 1672-1677.

Slansky, J.E., Y. Li, W.G. Kaelin, and P.J. Farnham. 1993. A protein synthesis-dependent increase in E2F1 mRNA correlates with growth regulation of the DHFR promoter. Mol.
Cell. Biol. 13: 1610-1618.

Vojtesek, B., J. Bartek. C.A. Midgley, and D.P. Lane. 1992. An immunochemical analysis of the human nuclear phosphoprotein p53. J. Immunol. Methods 151: 237-244.

Weinberg, R.A. 1995. The retinoblastoma protein and cell cycle control. Cell 81: 323-330.

Weintraub, S.J., C.A. Prater, and D.C. Dean. 1992. Retinoblastoma protein switches the E2F site from positive to negative element. Nature 358: 259-261.

Weintraub, S.J., K.N.B. Chow, R.X. Luo, S.H. Zhang, S. He, and D.C. Dean. 1995. Mechanism of active transcriptional repression by the retinoblastoma protein. Nature 375: 812-815.

Wu, C.-L., L.R. Zukerberg, C. Ngwu, E. Harlow, and J.A. Lees. 1995. In vivo association of E2F and DP family proteins. Mol. Cell. Biol. 15: 2536-2546.

Wu, C.-L., M. Classon, N. Dyson, and E. Harlow. 1996. Expression of dominant-negative mutant DP-1 blocks cell cycle progression in G1. Mol. Cell. Biol. 16: 3698-3706.

Wu, X.W. and A.J. Levine. 1994. p53 and E2F-1 cooperate to mediate apoptosis. Proc. Natl. Acad. Sci. 91: 3602-3606.

Yamasaki, L., T. Jacks, R. Bronson, E. Goillot, E. Harlow, and N. Dyson. 1996. Tumor induction and tissue atrophy in mice lacking E2F-1. Cell 85: 537-548.

Zamanian, M. and N.B. La Thangue. 1992. Adenovirus Ela prevents the retinoblastoma gene product from repressing the activity of a cellular transcription factor. EMBO I. 11: 26032610.

Zhu, L., S. Vandenheuvel, K. Helin, A. Fattaey, M. Ewen, D. Livingston, N. Dyson, and E. Harlow. 1993. Inhibition of cell proliferation by $\mathrm{p} 107$, a relative of the retinoblastoma protein. Genes \& Dev. 7: 1111-1125. 


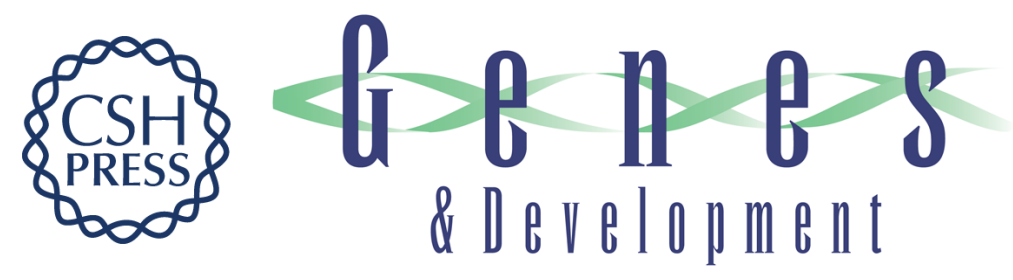

\section{Induction of DNA synthesis and apoptosis are separable functions of E2F-1.}

A C Phillips, S Bates, K M Ryan, et al.

Genes Dev. 1997, 11:

Access the most recent version at doi:10.1101/gad.11.14.1853

References This article cites 48 articles, 22 of which can be accessed free at:

http://genesdev.cshlp.org/content/11/14/1853.full.html\#ref-list-1

License

Email Alerting

Service

Receive free email alerts when new articles cite this article - sign up in the box at the top right corner of the article or click here.

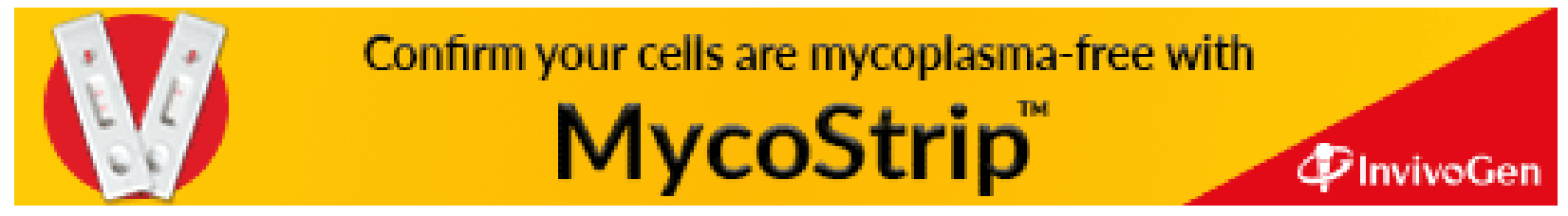

